\title{
Fall 2 - 38 Jahre, weiblich, rezidivierende Harnwegsinfektionen
}

In die urologische Praxis kommen immer wieder "auswärtige“ Patienten mit Beratungswunsch. Dabei handelt es sich um Erkrankte, die bereits bei Ärzten anderer Fachrichtung in Behandlung sind. Insbesondere Patientinnen mit rezidivierenden Harnwegsinfektionen und derzeitiger oder auch vorheriger Therapie durch den Gynäkologen oder Hausarzt gehören dazu. Auch für sie können urologische Wahlleistungen sinnvoll sein.

E ine häufig vorkommende Situation in - der urologischen Sprechstunde ist die Beratung einer gesunden Frau, die primär durch eine andere Arztpraxis betreut wird und sich mit einer Überweisung oder auf eigene Initiative aufgrund rezidivierender Harnwegsinfektionen (HWI) vorstellt. Die HWI wird sehr häufig klinisch diagnostiziert und insuffizient therapiert. In nicht urologischen Praxen erfolgen bei akuten Zystitiden in den seltensten Fällen eine mikrobiologische Untersuchung des Urins und eine Resistenzbestimmung.

Bei der zu Anfang beschriebenen $\mathrm{Pa}-$ tientin wurde vor dem Besuch in der urologischen Praxis noch keine Sonografie des Harntrakts durchgeführt. Insgesamt empfindet die Patientin ihre Situation als sehr belastend und möchte über potenzielle Risikofaktoren und Prophylaxemöglichkeiten aufgeklärt werden. Einen breiten Raum nimmt entsprechend die Besprechung von Vorteilen und Risiken der verschiedenen Formen der Rezidivprophylaxe ein.

\section{Leistungsangebot im konkreten Patientenfall}

Bei der Patientin, bei der eine HWI, eine wiederkehrende oder auch eine chronisch rezidivierende HWI bestätigt oder ausgeschlossen werden sollen, muss eine gründliche Anamnese, auch hinsichtlich des gynäkologischen Zustands, von Symptomen, Befunden und Risikofaktoren erhoben werden.

Gemäß der S3-Leitlinie zu HWI kann auf eine Urinuntersuchung und weitergehende Diagnostik verzichtet werden [1]. Sehr viele urologische Facharztpraxen werden aber trotzdem eine Urinuntersuchung veranlassen, um eine asymptomatische Bakteriurie und eine HWI zu differenzieren.
Die Anspruchshaltung und der Beratungsbedarf der Patientin mit HWI, die zur Zweitmeinungsberatung kommt, sprengen deutlich den Rahmen der allgemeinen Sprechstunde. Der Arzt sollte sofort offen kommunizieren, dass die gewünschte Beratung und anschließende Untersuchung inklusive der sinnvollerweise zugehörigen Sonografie des Harntrakts keine Leistungen sind, die durch die Gesetzliche Krankenversicherung (GKV) abgedeckt werden. Folgerichtig und berufsrechtlich korrekt ist daher, die erweiterte Diagnostik nach der Gebührenordnung der Ärzte (GOÄ) $\mathrm{zu}$ liquidieren.

Vor der Zweitmeinungsberatung ist die schriftliche Einwilligung der Patientin zu dieser „privatärztlichen Behandlung“ zwingend notwendig. Der Arzt muss darauf hinweisen, dass die geplanten Untersuchungen außerhalb der GKV und nach der Gebührenordnung für Ärzte (GOÄ) erbracht werden. Zu-

Tab.1: Abrechnungsbeispiele für Wahlleistungen bei Patientinnen mit wiederkehrenden Harnwegsinfektionen

GOÄ-Ziffer Legende

Beratung/Untersuchung

1 oder 3 , je nach Aufwand Beratung

5 oder 6 Untersuchung

$410,420,420$ Ultraschall

Immunisierung gegen Enterobakterien

5

5

375 Beratung Untersuchung

GÖ̈ = Gebührenordnung für Ärzte

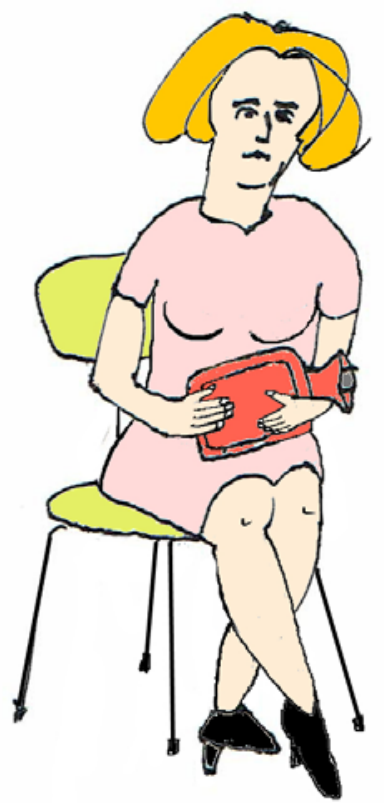

dem muss er benennen, welche Kosten dabei entstehen.

\section{Fazit}

Gemäß der S3-Leitlinie zu HWI bei Erwachsenen gilt die Sonografie der Nieren und Harnwege als primäre bildgebende Diagnostik, um komplizierende Faktoren abzuklären. Ich berate meine Patientin eingehend über die Vorteile und den Gewinn an Sicherheit durch diese Sonografie. Eine routinemäßige Zystoskopie ist bei ansonsten gesunden Frauen mit rezidivierenden HWI nicht indiziert.

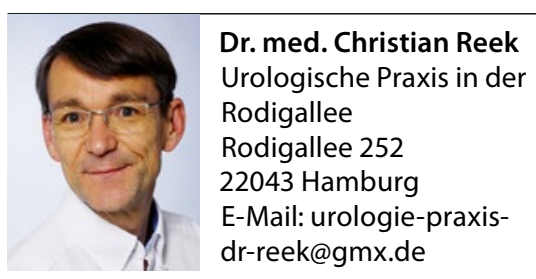

\section{Literatur}

1. Leitlinienprogramm Onkologie (Deutsche Krebsgesellschaft, Deutsche Krebshilfe, AWMF). S3-Leitlinie Epidemiologie, Diagnostik, Therapie und Management unkomplizierter bakterieller ambulant erworbener Harnwegsinfektionen bei erwachsenen Patienten, Langfassung 4.0, 2010 AWMFRegisternummer: 043/044, http://www. awmf.org/uploads/tx_szleitlinien/043-044I_ S3_Harnwegsinfektionen_2010-abgelaufen. pdf (Zugriff am: 11.2.2017). Abgelaufen, Leitlinie wird z. Zt. überprüft. 